\title{
Weakly Tapered Silicon Nanopillar Resonators with Spatially Well-Separated Whispering Gallery Modes for Si-based Lasers
}

Myunghae Seo ${ }^{a}$, Kihyun Kim ${ }^{a}$, Hyeonsu Cho ${ }^{a}$, Sol Yoon ${ }^{b}$, Byoung Don Kong ${ }^{b,}{ }^{*}$, M. Meyyappan ${ }^{c}$ and Chang-Ki Baek ${ }^{a, b, *}$

${ }^{a}$ Department of Creative IT Engineering and Future IT Innovation Lab., Pohang University of Science and Technology (POSTECH), Pohang 37673, Republic of Korea

${ }^{\mathrm{b}}$ Department of Electrical Engineering, Pohang University of Science and Technology (POSTECH), Pohang 37673, Republic of Korea

c NASA Ames Research Center, Moffett Field, California 94035, USA
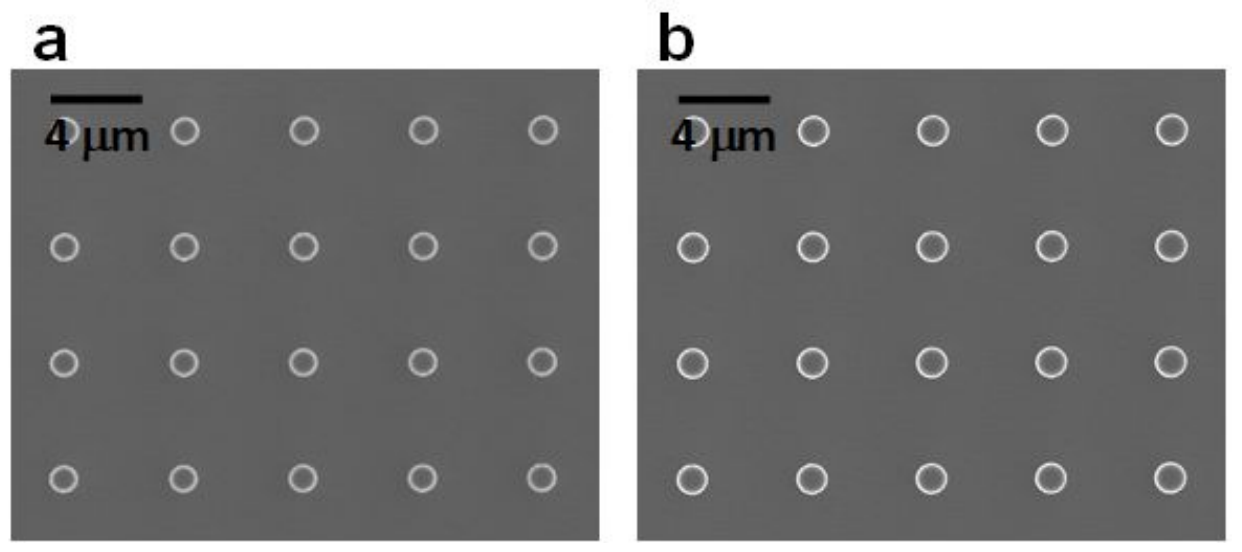

Figure S1. Top view SEM images of fabricated asymmetric SiNPs. (a) Negative SiNPs. (b) Positive SiNPs. Each SiNP has a spacing of $4 \mu \mathrm{m}$ to avoid optical interference from adjacent pillars. The scale bars represent $4 \mu \mathrm{m}$. 


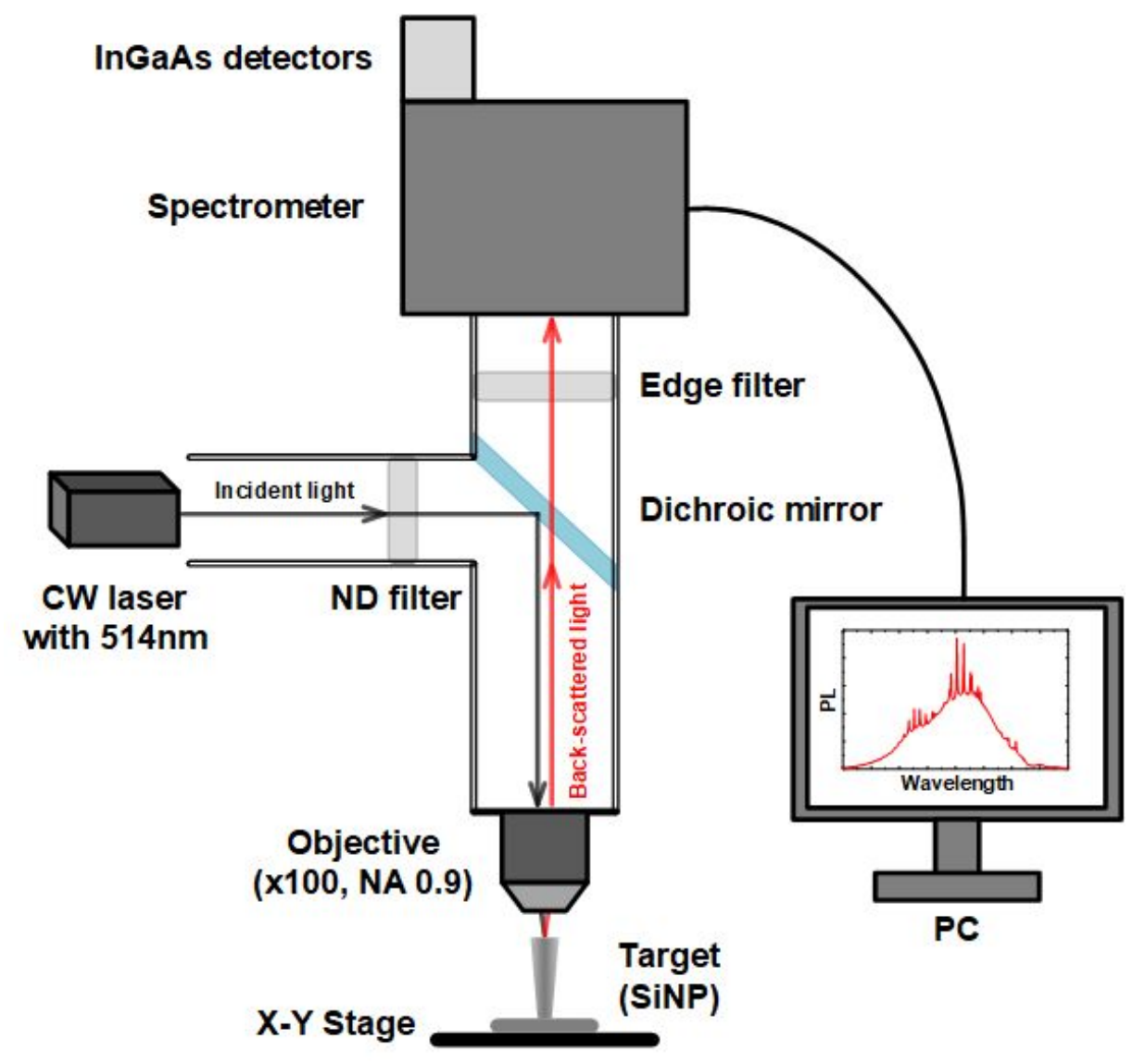

Figure S2. Schematic of the confocal PL measurement system. 


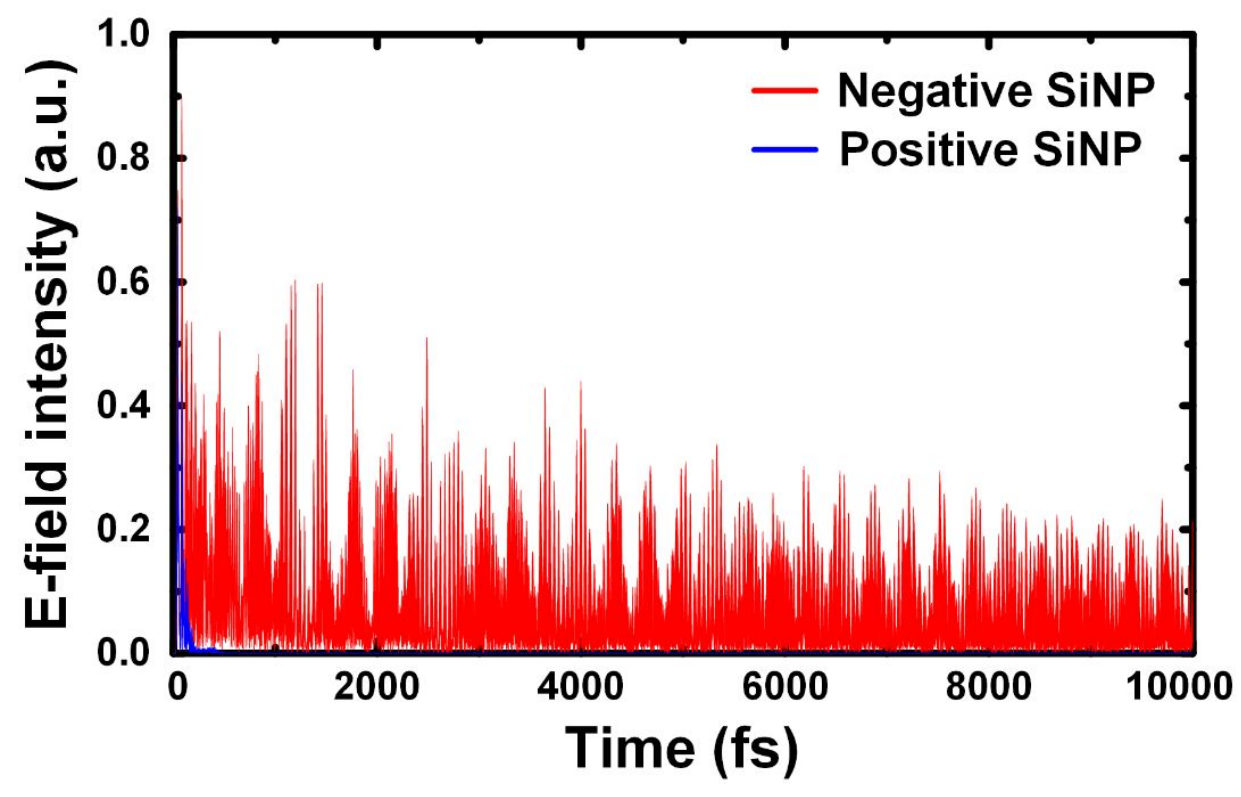

Figure S3. The electric field intensity over time from the asymmetric SiNPs. The time signals were recorded until the end of the simulations using a time monitor located near the top facet of the SiNPs. After the same amount of incident light was applied, the negative SiNP maintained WGMs well up to $10000 \mathrm{fs}$, whereas the positive SiNP did not form any WGM in which the electric field intensity rapidly converged to zero. 


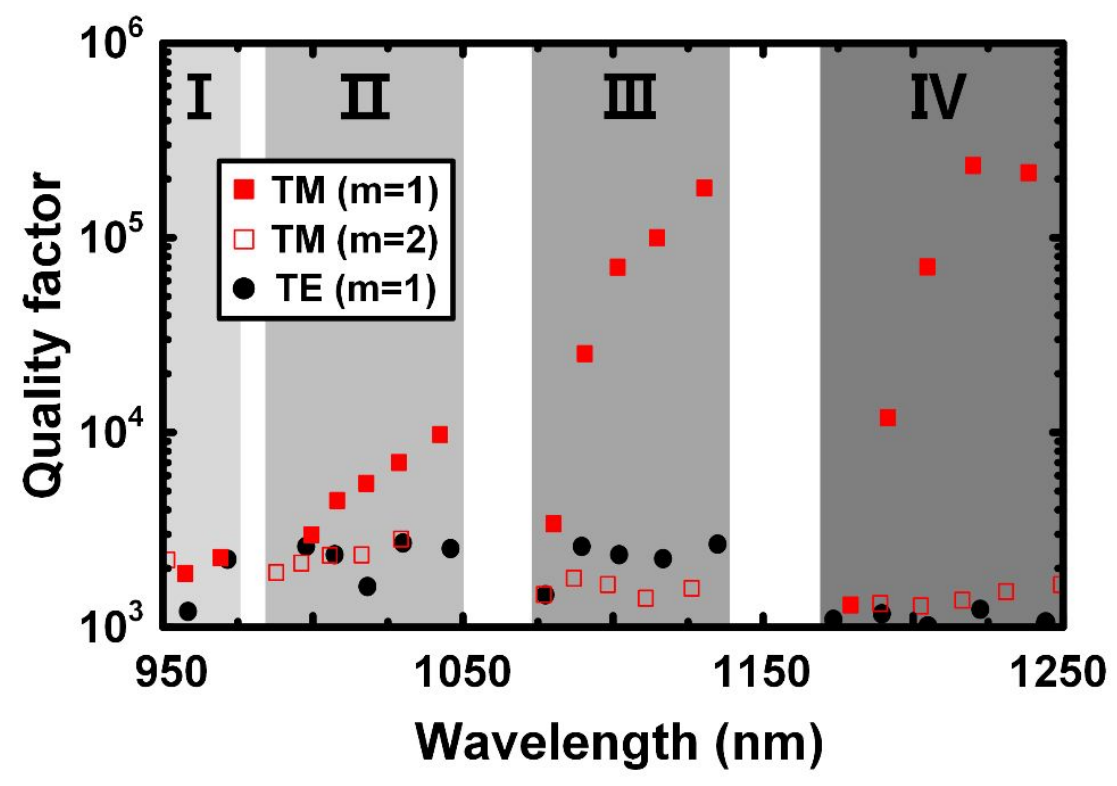

Figure S4. Quality factor $(Q)$ analysis in negative SiNP using FDTD. TM $(\mathrm{m}=1)$ exhibited a relatively high $Q$ compared to other modes, and $Q$ reached its maximum at wavelengths higher than the band edge of silicon $(\sim 1120 \mathrm{~nm})$ due to the very low extinction coefficient (near zero $^{\mathrm{S} 1}$ ). The magnitudes of $Q$ were higher than the experimental results because the non-radiative effects caused by the indirect bandgap of Si were not fully represented in the simulations. The modes with shorter wavelength in each region, $Q$ was relatively low, and this is because the light was not confined strongly at the bottom of the negative SiNP. 


\section{High $Q$ calculations in time signal using FDTD}

As observed in Figure S3, the E-field intensities in negative SiNPs survived till the end of the simulation time. Due to the high $Q$ and low loss, the simulation until the intensity completely decays is impractical. Since the FWHM bandwidth of each mode is affected by the simulation time, it is incorrect to calculate $Q$ as $Q=\lambda_{0} / \Delta \lambda$ in the frequency domain. If the time domain signal is not completely decayed, $Q$ can be calculated by estimating the rate of energy decay in the time domain $^{\text {S2 }}$ by using the following equation.

$$
Q=\frac{-2 \pi f_{R} \log _{10}(e)}{2 m}
$$

where $f_{R}$ is the resonant frequency of mode, and $m$ is the slope of the log of time signal envelope. 
a
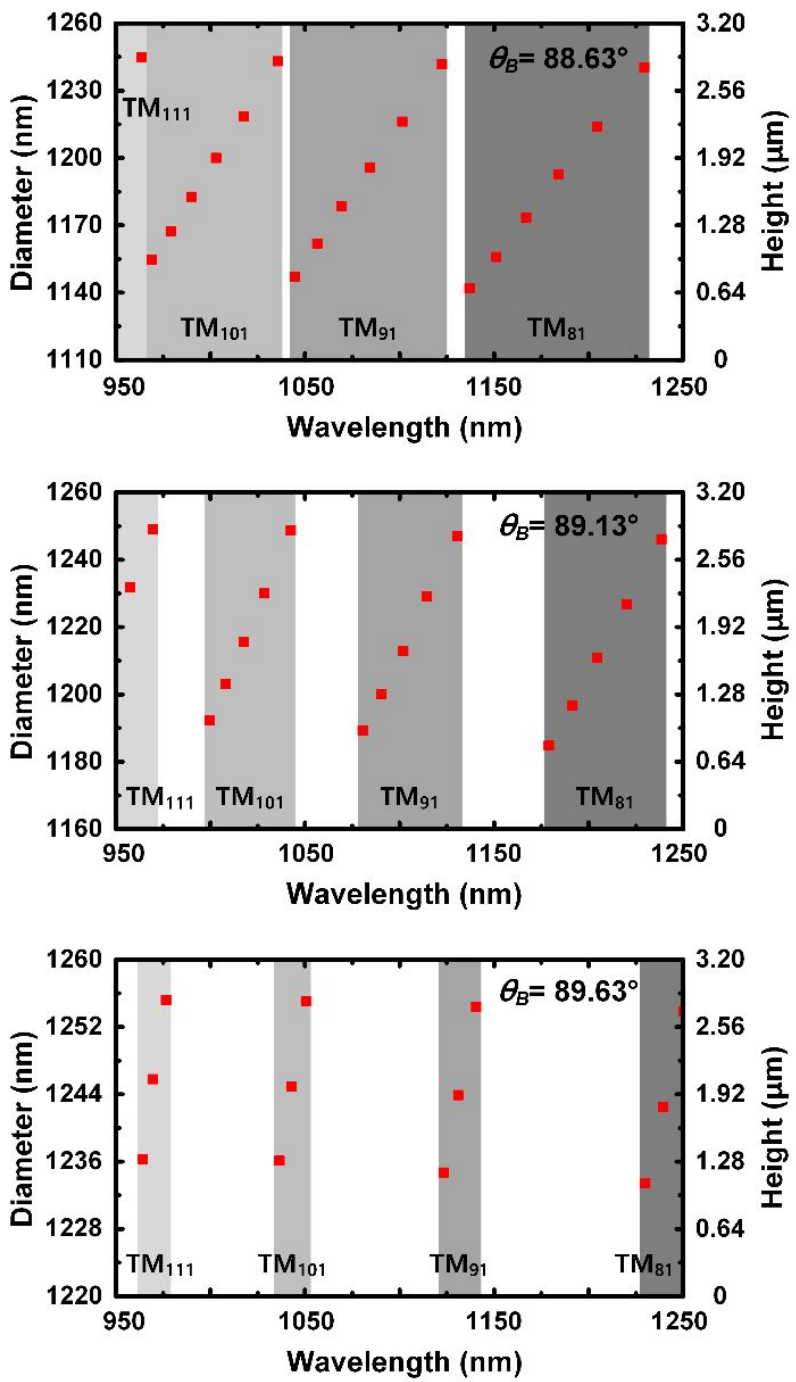

b
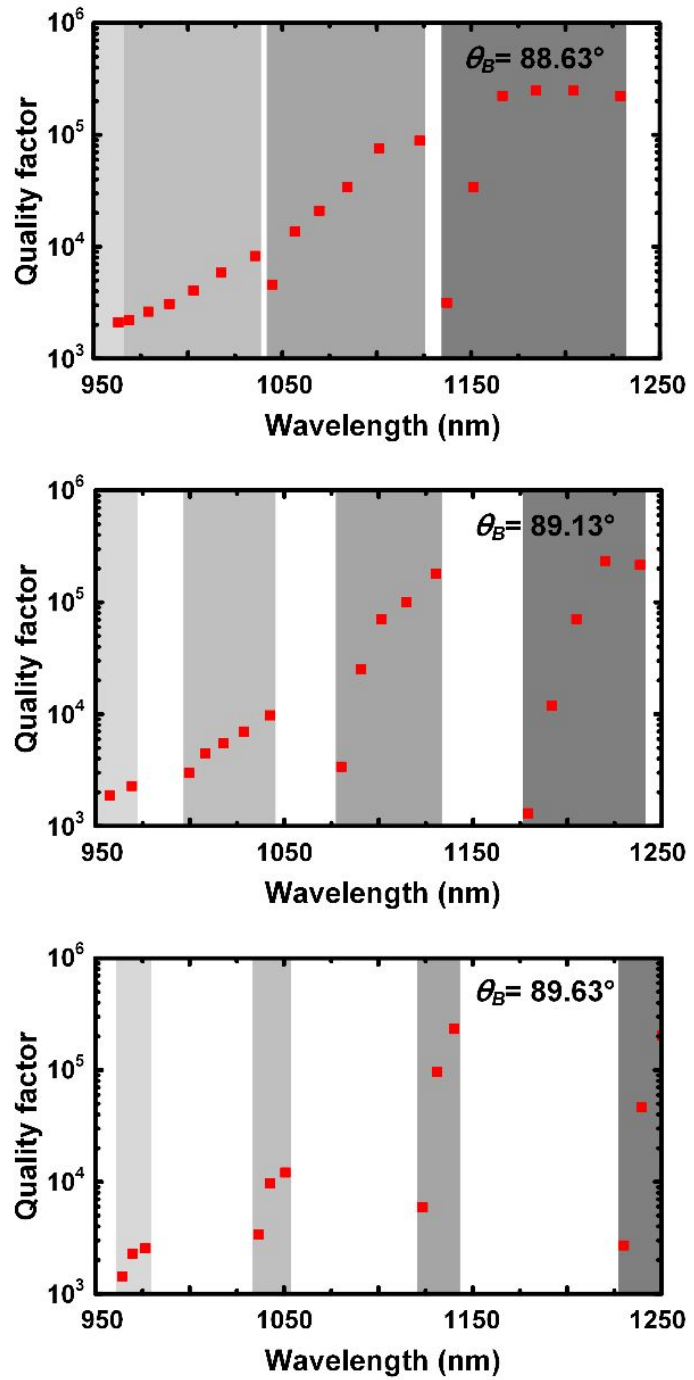

Figure S5. Slope dependence of $\mathrm{TM}(\mathrm{m}=1)$ in negative SiNPs. (a) Distributions of mode depending on the diameter and height of the SiNP. (b) $Q$ analysis. The value of $Q$ was hardly affected by the variation of $\theta_{B}$. Each mode was classified into four wavelength regions according to the azimuthal number $n$. 

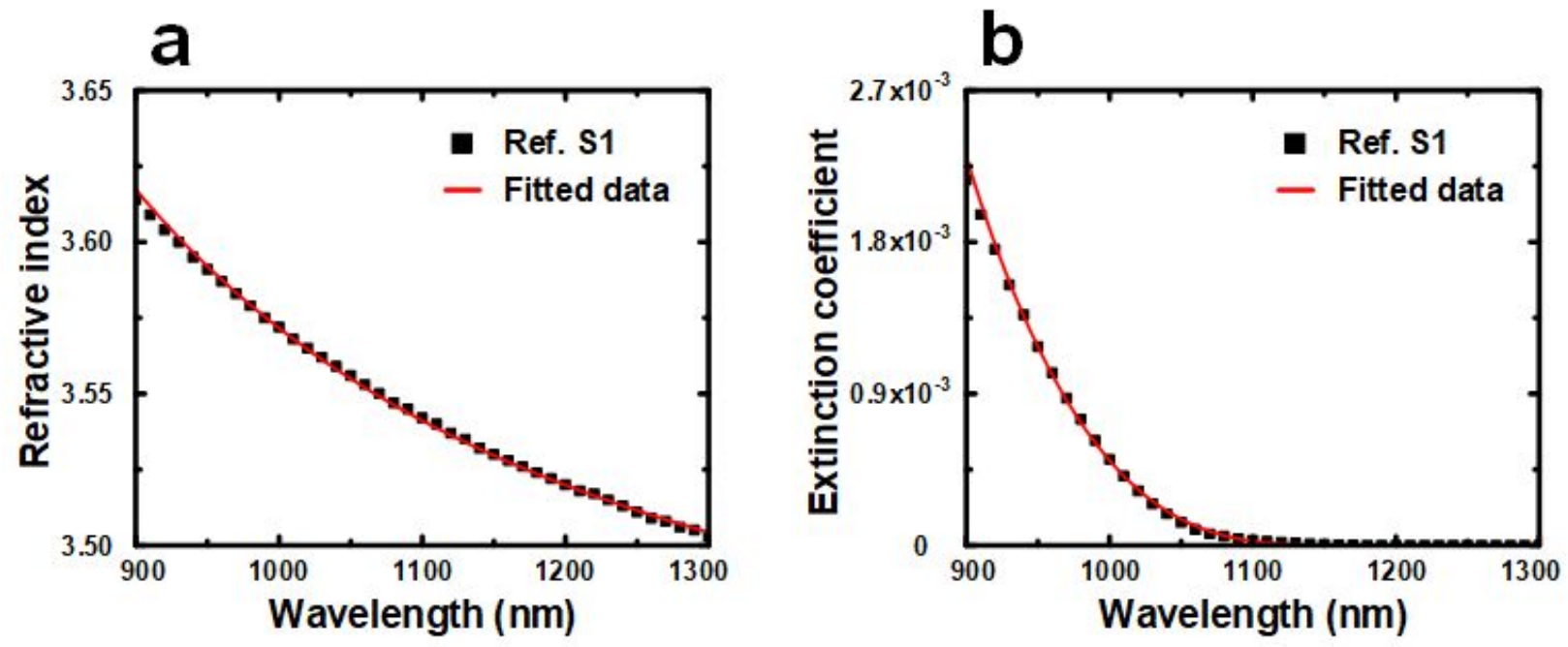

Figure S6. The complex refractive index of Si used in the optical simulations. (a) Refractive index. (b) Extinction coefficient. Black square: taken from ref. S1; Red line: fitted data.

\section{Verification of free spectral range (FSR)}

The experimental results show an $\mathrm{FSR}=85.14 \mathrm{~nm}$ with respect to the $1102.20 \mathrm{~nm}$ resonant peak. The FSR can be defined by the following equation ${ }^{\mathrm{S} 3}$ :

$$
F S R=\lambda_{r e s}^{2} / n_{g} \pi D_{r e s}
$$

where $\lambda_{\text {res }}$ is the resonant wavelength of mode, $\mathrm{n}_{\mathrm{g}}$ is the group refractive index, and $D_{\text {res }}$ is the diameter of the resonator. The optical simulation showed excellent agreement with the experimental results: FSR $=84.08 \mathrm{~nm}$ at $\lambda_{\text {res }}=1101.60 \mathrm{~nm}$ and $D_{\text {res }}=1212.80 \mathrm{~nm}$ at which the mode was observed. Based on these values, the calculated group index $n_{g}=3.7881$ is almost the same as extracted group index $n_{g}=3.8070$ from Figure S6. Therefore, our experimental and simulation results are reliable. 


\section{REFERENCES}

1. Green, M. A. Self-consistent optical parameters of intrinsic silicon at $300 \mathrm{~K}$ including temperature coefficients. Sol. Energy Mater. Sol. Cells 2008, 92, 1305-1310.

2. Chrostowski, L.; Hochberg, M. Modelling And Design Approaches. In Silicon Photonics Design: From Devices to Systems; Cambridge University Press: Cambridge, 2015; pp $28-46$.

3. Rabus, D. G. Integrated Ring Resonators: The Compendium; Springer: Berlin, 2007; Vol. 127. 